\title{
«La porte de derrière ». Sodomie et incrimination politique : des caricatures contre Cambacérès (1814-1815)
}

"The Back Door ». Sodomy and political incrimination: caricatures against

Cambacérès (1814-1815)

Emmanuel Fureix

\section{(2) OpenEdition}

Journals

Édition électronique

URL : https://journals.openedition.org/ahrf/11704

DOI : $10.4000 /$ ahrf. 11704

ISSN : 1952-403X

Éditeur :

Armand Colin, Société des études robespierristes

Édition imprimée

Date de publication : 1 septembre 2010

Pagination : 109-130

ISBN : 978-2-200-92633-5

ISSN : 0003-4436

Référence électronique

Emmanuel Fureix, « «La porte de derrière ». Sodomie et incrimination politique : des caricatures contre Cambacérès (1814-1815) », Annales historiques de la Révolution française [En ligne], 361 | juillet-

septembre 2010, mis en ligne le 01 septembre 2013, consulté le 23 avril 2022. URL : http:// journals.openedition.org/ahrf/11704; DOI : https://doi.org/10.4000/ahrf.11704 


\title{
"LA PORTE DE DERRIÈRE ». SODOMIE ET INCRIMINATION POLITIQUE : DES CARICATURES CONTRE CAMBACÉRÈS (1814-1815)
}

Emmanuel FUREIX

\begin{abstract}
La caricature à connotation sexuelle, dans le cadre d'une campagne ad hominem, peut devenir une arme politique en temps de crise. En 1814, au retour des Bourbons, l'ancien archichancelier d'Empire, Cambacérès, fait ainsi l'objet d'une satire graphique sans précédent. Une vingtaine de caricatures représentent, plus ou moins allusivement, ses penchants « supposés » de sodomite. Une rumeur mondaine devient un stigmate public, qui vise à empêcher toute reconversion dans le nouveau régime. Ce dispositif politique efficace repose sur la figuration d'un corps réduit au double péché de luxure et de gourmandise. L'article examine plus précisément les usages graphiques de la sodomie dans une incrimination politique, et étudie la présence dans la caricature d'un imaginaire commun de la sodomie, à un âge où la spécification de I'homosexualité n'est pas réalisée. II souligne, par l'analyse de sources policières et judiciaires, les effets politiques de ce dispositif, et ses contradictions à un moment où tente de s'imposer une civilité des mœurs politiques.
\end{abstract}

Mots-clés : caricatures, Cambacérès, homosexualité, sodomie, imaginaires sociaux, exclusion politique.

Sur une caricature relative au Congrès de Vienne, «La restitution ou chaqu'un son compte» (fig. 45), les monarques européens se partagent autour du tsar Alexandre les dépouilles de l'Empire napoléonien. En marge de cette scène, un Cambacérès bedonnant, accompagné de deux 
acolytes ${ }^{1}$, emprunte discrètement la « porte de derrière », tout en proclamant « Je connais cette porte». Par ce calembour, le caricaturiste anonyme désigne tout à la fois une inclination sexuelle et une mise à l'écart de la vie politique à la faveur de la Restauration. Il condense efficacement les mécanismes d'une campagne satirique et politique orchestrée à la chute de l'Empire contre l'ancien archichancelier de Napoléon : le déposséder de toute fonction publique en dévoilant ses scandaleux penchants de « sodomite ».

Les usages politiques du sexe par la caricature renvoient à l'émergence graduelle au XVIII ${ }^{\mathrm{e}}$ siècle de l'opinion publique comme instance de jugement. Ils rappellent les liens étroits entre politique et pornographie avant et pendant la Révolution, et le travail de sape des fondements traditionnels du pouvoir ${ }^{2}$. Estampes et pamphlets ont alors conjugué leurs effets pour désacraliser un corps royal impuissant ${ }^{3}$, et dénoncer la « tribade » Marie-Antoinette ${ }^{4}$ ou, plus marginalement, le « bougre » marquis de Villette ${ }^{5}$. Le dévoilement de pratiques «contre-nature» ou d'excès orgiaques visait à dénoncer la dégénérescence ou la trahison des élites. Il incarnait aussi, dans un moment de régénération démocratique, la transparence idéale d'un corps politique où le citoyen devenait spectateur des rapports secrets de pouvoir. Il autorisait, en des circonstances exceptionnelles, le défoulement de fantasmes collectifs jusque-là souterrains. Il permettait enfin, en contrepoint, la construction sexuée d'une Révolution virile, centrée sur un ordre familial devenu " fraternel» après le régicide ${ }^{6}$.

(1) Il s'agit de d'Aigrefeuille et de Villevieille, personnages historiques de second plan, mais omniprésents dans le corpus de caricatures étudiées. Amis de Cambacérès depuis sa jeunesse montpelliéraine, ils lui restent proches jusqu'en 1814.

(2) Lynn Hunt, « Pornography and the French Revolution », in The Invention of Pornography. Obscenity and the Origins of Modernity, 1500-1800, New York, Zone Books, 1996, p. 301-340. Antoine de BAECQUE «The Livres remplis d'horreurs: Pornographic Literature and Politics at the Beginning of the French Revolution "), in Peter Wagner, ed., Erotica and the Enligthnement, Francfort, 1991.

(3) Antoine DE BAECQUe, Le corps de l'histoire. Métaphores et politique (1770-1800), Paris, Calmann Lévy, 1993, p. 45-98.

(4) Chantal Thomas, La reine scélérate. Marie-Antoinette dans les pamphlets, Paris, Le Seuil, 2008 ( $1^{\text {rè }}$ éd. 1989), p. 133-143; Annie Duprat, Marie-Antoinette. Une reine brisée, Paris, Perrin, 2006, p. 140 et suiv.

(5) Claude Langlois, La caricature contre-révolutionnaire, Paris, Presses du CNRS, 1988, p. 21. Voir également l'article de Thierry Pastorello dans la présente livraison.

(6) Lynn Hunt, Le roman familial de la Révolution française, Paris, Albin Michel, 1995. Le régicide-parricide aurait engendré selon l'auteure une société de frères, excluant rapidement les femmes au nom, notamment, des déviances sexuelles de la « mauvaise mère » Marie-Antoinette. 
En 1814-1815, avec la restauration d'une « monarchie paternelle », la naturalisation de la différence des sexes s'appuie désormais sur un ordre religieux. Les «bonnes mœurs » se veulent le signe d'un retour à l'ordre naturel et divin. Mais la fragilité des légitimités et des régimes successifs - deux Restaurations ponctuées d'un retour à l'Empire durant les Cent-Jours - donne d'autres ressorts à la politisation du sexe. La révélation d'une déviance sexuelle permet d'exclure définitivement un individu des affaires publiques, et de redessiner ainsi des lignes de partage en un temps de confusion où abondent girouettes et transfuges - de Talleyrand à Fouché7. Dans les caricatures contre Cambacérès, les fesses rebondies, les regards lubriques et autres jeux de mots grivois ne doivent pas tromper : derrière les cancans et la dérision se cache un dispositif politique efficace, capable de mettre hors-jeu un acteur incommode, prototype du « juriste technicien $»^{8}$. Où l'on retrouve, sur un autre mode, les ressorts de «l'art de la calomnie » observé par Robert Darnton au XVIII ${ }^{\mathrm{e}}$ siècle 9 .

On ne peut s'expliquer autrement la violente campagne ad hominem subie par Cambacérès au début de la Restauration. Au moment où une implacable "guerre des images" oppose essentiellement Louis XVIII à Napoléon ${ }^{10}$, figuré en ogre ou en tyran ${ }^{11}$, on est frappé par la singularité du corpus hostile à Cambacérès ${ }^{12}$. Une cinquantaine de caricatures anonymes - corpus très conséquent - visent en quelques mois l'ancien archichancelier de Napoléon - pour l'essentiel entre mai et octobre $1814^{13}$. Une majorité d'entre elles - trente-et-une - font référence à l' « homosexualité » supposée de Cambacérès, dont vingt-quatre, plus ou moins allusivement, à la sodomie. À une seule exception près ${ }^{14}$

(7) Pierre SERnA, La République des girouettes (1789-1815 et au-delà). Une anomalie politique : la France de l'extrême centre, Seyssel, Champ Vallon, 2005, p. 149-304.

(8) Patrick Logoras-Flavigny, « Cambacérès et Locke », AHRF, n 307, 1997, p. 105-115.

(9) Robert Darnton, Le Diable dans un bénitier. L'art de la calomnie en France, 1650-1800, Paris, Gallimard, 2010.

(10) Annie Duprat, "Une guerre des images. Louis XVIII, Napoléon et la France en $1815 »$, Revue d'histoire moderne et contemporaine, $\mathrm{n}^{\circ}$ 47-3, 2000, p. 486-504.

(11) Catherine Clerc, La caricature contre Napoléon, Paris, Promodis, 1985; Hans-Peter Mathis (dir.), Napoléon I ${ }^{e r} v u$ à travers la caricature, Zürich, Verlag, 1998.

(12) Champrenury, Caricatures sous la République, l'Empire et la Restauration, Paris, Dentu, 1877 , p. 324 et suiv.

(13) Les caricatures considérées, des eaux-fortes diffusées sous forme de feuilles volantes, ne sont pour la plupart pas datées. Certaines d'entre elles sont toutefois évoquées dans la presse et les rapports de police, permettant une datation approximative. L'ensemble forme un corpus homogène, tant par les techniques que par les registres de la charge. Ce corpus précède le bannissement de Cambacérès en janvier 1816 dans la cohorte des conventionnels régicides relaps.

(14) «Cambacérès et Mademoiselle Cuisot». BNF Ae25 RES Fol. M311350. 
(fig. 52), leur registre n'est pas à proprement parler pornographique, mais relève du persiflage sexuel, codé. Ces caricatures, rarement montrées, ont été jusque-là cantonnées au rang d'indices de l'« homosexualité » de Cambacérès ${ }^{15}$. Il est sans doute plus pertinent d'y lire les modalités du passage de la rumeur publique à l'incrimination politique. Comment le corps privé de Cambacérès devient-il politique? À quels imaginaires sociaux renvoie la satire de la sodomie, à un âge où - si l'on suit Michel Foucault - la « spécification » de l'homosexualité n'est pas encore constituée ${ }^{16}$ ? Et quels sont les effets proprement politiques de cette dégradation symbolique?

\section{Le corps retourné : de l'archichancelier à « Tante Urlurette »}

Printemps 1814. Cambacérès, archichancelier de l'Empire, dignitaire inamovible, participe très activement aux derniers actes d'un régime chancelant. Le 29 mars, après un Conseil de Régence, il quitte la capitale, à l'approche des troupes alliées. Il arrive à Blois le 2 avril, en compagnie de l'impératrice et du roi de Rome. Il reste fidèle à Napoléon jusqu'à son abdication le 6 avril, avant d'adhérer le 10 au gouvernement provisoire dirigé par Talleyrand. Il retourne à Paris le 14 avril où il signe son adhésion aux actes pris jusque-là par le Sénat. Il attend en vain sa nomination dans la nouvelle Chambre des pairs, en juin 1814. Au même moment, il fait verser 200 francs pour l'érection de la statue d'Henri $\mathrm{IV}^{17}$. Son retournement, quoique tardif et limité, le rend théoriquement disponible pour le nouveau régime, celui de Louis XVIII. Une disponibilité vite anéantie par une campagne ad hominem qui replace le grand dignitaire d'Empire dans la sphère privée.

Les caricaturistes retournent ainsi le corps politique de Cambacérès en un corps privé, sexualisé et stigmatisé, celui d'un sodomite désigné par

(15) Pierre Vialles, L'archichancelier Cambacérès (1753-1824) d'après des documents inédits, Paris, Perrin, 1908, p. 362-378; Jean-Louis BoRY, Les cinq girouettes ou Servitudes et souplesses de Son Altesse Sérénissime Le Prince Archichancelier de l'Empire Jean-Jacques Régis de Cambacérès Duc de Parme, Paris, Ramsay, 1979.

(16) « La sodomie - celle des anciens droits civil ou canonique - était un type d'actes interdits; leur auteur n'en était que le sujet juridique. L'homosexuel du XIX ${ }^{\mathrm{e}}$ siècle est devenu un personnage : un passé, une histoire et une enfance, un caractère, une forme de vie; une morphologie aussi, avec une anatomie indiscrète et peut-être une physiologie mystérieuse ». Michel Foucault, Histoire de la sexualité, t.1, La volonté de savoir, Paris, Gallimard, 1976, p. 59.

(17) Journal des débats politiques et littéraires, 27 juin 1814. 
le sobriquet de « tante Urlurette ». La charge repose moins sur la déformation physiognomonique du corps que sur son retournement par le dévoilement de choses supposées cachées. Au début du mois de juin 1814 paraît la caricature emblématique, "Ma tante Urlurette " (fig. 46, cahier couleur $)^{18}$. Le titre renvoie à la fois au topos de l'homosexuel efféminé19, et, pour les initiés, au vaudeville de Désaugiers représenté en 1806, dans lequel Urlurette incarnait une vieille fille ridicule, tante de l'héroïne principale $^{20}$. On reconnaît Cambacérès sous les traits d'une grosse femme ornée de fanfreluches, de dentelles et d'une traîne soulevée par son ami, le comte d'Aigrefeuille, énorme, portant une poularde embrochée et l'Almanach des gourmands. Cambacérès tient de sa main droite un éventail, et de sa main gauche un sac avec cette inscription : « Haine aux femmes. Comédie. Vaudeville », désignant de l'index une ville en proie aux flammes, Sodome, précédée d'une statue de sel. Cette caricature condense plusieurs sources de discrédit politique : l'excès de pompe, la gourmandise, le désordre sexuel et la chute, subliminale, d'un Empire décadent. Le tout articulé autour d'un même registre de satire mondaine à clef. La critique s'éclaire si l'on sait que l'archichancelier recevait à sa table, autour des mets les plus raffinés, les hôtes les plus prestigieux de l'empereur. Le gastronome Grimod de La Reynière, ami de Cambacérès et de son aréopage, avait dédié en 1803 son Almanach des gourmands à d'Aigrefeuille, représenté sur la caricature. L'allusion à Sodome se révélait plus proprement politique. Elle confirmait des bruits insistants depuis le Consulat, liés au célibat et aux fréquentations de Cambacérès. Si aucune preuve formelle ne peut être donnée de son « homosexualité $»^{21}$, il est indéniable qu'il passait pour " sodomite" dans la haute société parisienne ${ }^{22}$. Quoi qu'il en soit, l'allusion au châtiment biblique de Sodome fonctionne aussi comme métaphore de l'exclusion politique de l'homme public Cambacérès.

(18) Elle est décrite dans le Journal de Paris du 11 juin 1814.

(19) Le mot «tante» est déjà employé à la fin du XVIII ${ }^{\mathrm{e}}$ siècle, dans le discours policier comme dans le vocabulaire des « sodomites » eux-mêmes, avant de se diffuser au XIX ${ }^{\mathrm{e}}$ siècle, singulièrement dans le monde carcéral où il désigne un " pédéraste dit passif et efféminé » (Laure MuRAT, La loi du genre. Une histoire culturelle du troisième sexe, Paris, Fayard, 2006, p. 33).

(20) Ma tante Urlurette, ou le chant du coq, folie-vaudeville en un acte de Désaugiers, Paris, Barba, 1806.

(21) Laurence Chatel de Brancion, Cambacérès. Maître d'œuvre de Napoléon, Paris, Perrin, 2009, p. 52-55.

(22) Pierre Vialles, op. cit., p. 358-359. 


\section{Politique et sodomie}

La sodomie en caricature ne vise pas seulement l'honneur d'un homme, mais un système de gouvernement, voire une politique de la famille. Trois caricatures relient ainsi, sur un mode apocalyptique, la saignée démographique des guerres impériales et la sodomie comme acte contre-nature. Cambacérès n'est que le double - inverti - de Napoléon. Une vignette-médaillon, réversible, représente ainsi les profils croisés de Napoléon et de Cambacérès, entourés de cette inscription : «Haine aux hommes, haine aux femmes, avec eux la fin du monde $»^{23}$. Deux autres caricatures intitulées "La fin du monde» précisent la charge. La première (fig. 47) représente sur un piédestal un buste à deux têtes - Napoléon et Cambacérès. Le buste de Napoléon, en forme de globe terrestre, est accompagné de flammes, de boulets et de canons. Le piédestal, orné d'instruments de torture, comporte l'inscription : "Invadere et evertere " («Envahir et anéantir »). Du côté de Cambacérès, sous un livre de "physique élémentaire » déchiré, un homme abat à coups de hache une statue de déesse-mère, en vertu de la devise : "Nunquam procreare » (« Ne jamais procréer »). L'autre caricature (fig. 48, cahier couleur), de même titre et de même esprit, représente d'un côté Napoléon exterminant, incendiant, massacrant, et de l'autre Cambacérès tournant ostensiblement le dos à trois jeunes femmes - attitudes également contraires à la perpétuation de l'humanité... La perversion d'un mode de gouvernement des hommes est associée à la transgression des normes sexuelles - procédé propre à la pornographie politique. Les plaisirs antiphysiques de l'archichancelier et les pulsions guerrières de l'ogre Napoléon sont ainsi placés sur le même plan. Il est vrai que l'archichancelier avait à sa façon contribué à l'effort de guerre voulu par Napoléon. Il avait chaque année pris la parole devant le Sénat conservateur pour appuyer les levées de conscrits, de 1806 à $1814^{24}$. Il avait également exercé, en l'absence de l'empereur, une partie du pouvoir exécutif, en 1813-1814. On peut aussi, peut-être, lire dans ces caricatures une critique du droit du mariage et de la famille imposé par le Code civil de 1804, dont Cambacérès fut l'un des rédacteurs. Le mariage, quoique tendu vers la

(23) BNF, Cabinet des estampes. N2 fol. Portraits. Parme (Jean-Jacques Régis de Cambacérès), vol. 1446.

(24) Pierre Vialles, op. cit., p. 315-318. 
procréation $^{25}$, y est contractualisé, et à ce titre, dissoluble. Or, aux yeux des royalistes intransigeants, l'institution du divorce, perpétuée par le Code civil, viole les lois naturelles de la famille et sape les fondements de la monarchie, ainsi que l'avait dénoncé Bonald dès $1801^{26}$.

Par ailleurs, la métaphore de l'ogre (ou du minotaure), si prégnante dans la caricature anti-napoléonienne ${ }^{27}$, est sexualisée autour de Cambacérès. Ce dernier est régulièrement présenté en chasseur de jeunes hommes, la pédérastie redoublant symboliquement la conscription forcée. Dans « Le grand Dardanus et ses capitaines de recrutement » (fig. 49, cahier couleur), on peut voir Cambacérès, nu sous sa toge, tenir une énorme fourchette en guise de sceptre et observer avec satisfaction un éphèbe aux formes suggestives. D'Aigrefeuille apprête le jeune soldat, tandis que Villevieille, autre compagnon de Cambacérès, se lèche les doigts. Les bustes d'Adrien et d'Antinoüs délivrent la clef sexuelle de l'image, renforcée par le titre - qui évoque moins un préfet du prétoire des Gaules du v viècle, Caius Postumus Dardanus, qu'un calembour grivois, " dard-anus ». La gourmandise notoire du cercle de Cambacérès permet de jouer sur les trois registres de la cuisine, de la pédérastie et de la tératologie politique. On retrouve cette conjonction dans plusieurs caricatures analogues ${ }^{28}$, et tout particulièrement dans le "serment des voraces » (fig. 50, cahier couleur), qui repose sur le détournement parodique de la toile de David Le Serment des Horaces (1784), détournement déjà pratiqué sous la Révolution et supposant une connivence culturelle avec le public. Les trois Horaces sont ici composés de Villevieille et d'Aigrefeuille, et d'un éphèbe enrôlé par les deux compères. Le père des Horaces est incarné par Cambacérès, les trois glaives du tableau de David se sont métamorphosés en fourchettes, redoublées par trois poulardes et trois bouteilles. À droite, l'héroïne tragique Camille est figurée par l'amante de façade de Cambacérès, l'actrice Cuisot, fort délaissée

(25) Portalis, autre rédacteur du Code civil, définissait le mariage en ces termes : «Le mariage est la société de l'homme et de la femme unis pour perpétuer leur espèce, pour s'aider, par des secours mutuels, à porter le poids de la vie, et pour partager leur commune destinée ».

(26) « Le divorce est directement contradictoire à l'esprit et aux principes de la monarchie héréditaire ou indissoluble » (Louis de BONALD, Du divorce considéré au XIX siècle relativement à l'état domestique et à l'état public de société, Paris, Leclère, 1805 [1 ${ }^{\text {ère }}$ éd. 1801], p. 68).

(27) Voir notamment Napoléon... Aigle ou ogre? Catalogue de l'exposition du 11 décembre 2004 au 2 décembre 2005 au musée de l'Histoire vivante de Montreuil.

(28) «Quel friand morceau pour un gourmand», 20 juillet 1814, Beauplé père, rue St Sévrin $n^{\circ} 18$ (BNF Collection de Vinck, $n^{\circ} 9331$ ), et « Le retour de la chasse aux culs blancs, ou les bons chiens d'arrêt » (BNF N2 fol. Portraits. Parme - Jean-Jacques Régis de Cambacérès -, vol. 1446). 
dans ce monde très masculin, et qui exprime ainsi son dépit : " Il m'en cuit, sotte que je suis ».

Également fondée sur le registre parodique, la caricature «Les lutteurs » (fig. 51) détourne un groupe statuaire antique (« les pancratiastes $\gg)^{29}$ pour représenter un conflit politique entre Napoléon et Cambacérès, tout en évoquant implicitement l'« homosexualité »de l'archichancelier. On y voit Napoléon, dans un surprenant corps à corps, chevaucher et éperonner l'archichancelier, non loin du trône impérial, sous le regard de la foule présente dans la cour des Tuileries. Cambacérès s'agrippe en vain à un document que l'on suppose être le décret d'ajournement du Corps législatif, auquel il s'était opposé avec succès jusqu'en $1813^{30}$. Une relation politique est sexualisée - ici allusivement - suivant en cela des précédents fournis par la pornographie politique révolutionnaire $^{31}$. Cambacérès, connu pour son influence décisive sur l'empereur, est ici en position passive, doublement disqualifiante.

\section{Des bruits publics à la caricature}

La caricature métaphorise la sodomie en mode de gouvernement. Mais plus simplement, et plus fréquemment au sein du corpus, elle met en image des rumeurs diffusées depuis l'Empire voire le Consulat ${ }^{32}$, dévoilant la «vie privée » supposée de Cambacérès. La publication et l'exposition de ces caricatures transforment la rumeur en stratégie diffamatoire. Distinguée de la calomnie en ce qu'elle ne suppose pas nécessairement le mensonge, la diffamation, encore non régulée en 1814, porte atteinte à «l'honneur » ou à la «considération » de la personne visée, à sa fama publica $^{33}$. Ces caricatures diffamatoires, dans l'histoire de la

(29) Philippe Magnier avait réalisé une copie des Lutteurs (original grec perdu du $\mathrm{III}^{\mathrm{e}}$ siècle av. J.-C., copie romaine du $\mathrm{I}^{\mathrm{er}}$ siècle) vers 1684 . Ce groupe statuaire avait été exposé à Marly avant la Révolution, puis aux Tuileries à partir de 1797. C'est sans doute cette statue que connaissait le caricaturiste.

(30) Interprétation proposée par Laurence CHATEL DE BRANCION (op. cit., p. 594).

(31) Songeons ainsi aux "Torts de Necker envers la France », caricature représentant Necker en train de sodomiser une allégorie de la France (Lynn Hunt, The Invention [...] op. cit., p. 320).

(32) Voir Bonaparte et les Bourbons. Relations secrètes des agents de Louis XVIII à Paris sous le Consulat (1802-1803), publiées avec une introduction et des notes par le $C^{\text {te }}$ Remacle, Paris, Perrin, 1899.

(33) La loi de Serre de 1819 « sur la répression des crimes et des délits commis par la voie de la presse ou par tout autre moyen de publication » définit la diffamation comme « toute allégation ou imputation d'un fait qui porte atteinte à l'honneur ou à la considération de la personne ou du corps auquel le fait est imputé ». 
satire graphique de la Restauration, détonnent. Les caricatures politiques visent alors beaucoup plus la trahison - celle des fameuses " girouettes » - ou l'anachronisme politique - celui de l'émigré "Monsieur de la Jobardière $»^{34}$ ou des célèbres «chevaliers de l'ordre de l'éteignoir » - que la corruption des mœurs.

Parmi les rumeurs mises en image, figure la relation très particulière entre Cambacérès et une actrice du théâtre des Variétés, Henriette Cuisot. Sous l'Empire cette relation avait été construite comme un contrefeu aux rumeurs sur l' " homosexualité » de Cambacérès ${ }^{35}$. Un dialogue de Cour, non attesté, circula à son propos. À son interlocuteur, qui le félicitait de la grossesse de $\mathrm{M}^{\text {elle }}$ Cuisot, Cambacérès aurait répondu : "Cela regarde M. de R., car je ne l'ai connue que postérieurement $»^{36}$. C'est cette réplique savoureuse qui semble figurée littéralement dans la charge pornographique «M. Cambacérès et $\mathrm{M}^{\text {elle }}$ Cuisot » (fig. 52), où l'archichancelier sodomise la jeune actrice, qui lui déclare : "Vous me tourmentez comme un remède, Monseigneur ». La caricature confine ici à l'estampe libertine, en une scène de bordel renforcée par le jeu de miroir dévoilant les fesses de Cambacérès. Le tribunal de l'opinion semble pénétrer l'alcôve d'un homme d'État dont les dérèglements sont ainsi exhibés. Plus allusive mais assez proche, la « Petite loge, ou l'archifou » (fig. 53), alors jugée indécente, représente Cambacérès au théâtre des Variétés, observant et applaudissant $\mathrm{M}^{\text {elle }}$ Cuisot, jouant du fifre. Représentée de dos, habillée en homme, elle offre son postérieur au regard concupiscent de l'archichancelier. Une caricature en abîme, affichée sur la scène, renvoie à «Tante Urlurette » et rappelle l'identité sodomite de Cambacérès. La rumeur mondaine, confinée aux cercles avertis, devient ainsi publique et politique à travers le prisme de la caricature. Un mot d'esprit est mis en image et en récit, ainsi que le confirme une autre caricature au titre

(34) Emmanuel DE WARESQUIEL, «La caricature antinobiliaire sous la Restauration. De l'émigration à l'ultracisme (1814-1820)», dans Annie Duprat, Michèle MénARd (dir.), Histoire, images, imaginaires (fin $X V^{e}$ siècle-début $X X^{e}$ siècle), Le Mans, Université du Maine, 1998, p. 427-440.

(35) Fouché, ministre de la Police, écrit ainsi dans un rapport destiné à l'Empereur, le 24 mai 1808 : « On dit que l'archichancelier conduit de front trois intrigues. À l'Opéra, une demoiselle Athalie, danseuse ; aux Français Melle Rose Dupuis; aux Variétés la demoiselle Cuisot. L'archichancelier prenant trois femmes pour prouver qu'on l'accuse à tort de ne pas les aimer ne ressemble pas mal à ces aristocrates de 93 dont les portefeuilles étaient pleins de cartes de sûreté et de certificats de civisme » (cité par Laurence Chatel DE BRANCION, op. cit., p. 470).

(36) Pierre Vialles, op. cit., p. 360. 
éloquent, «Le mariage de M. Lagobbe et de $\mathrm{M}^{\text {elle }}$ Retournée ${ }^{37}$. On reste là assez proche des logiques de persiflage de Cour, de satire à clef et de jeux de mots.

La figure de Cambacérès s'est prêtée à ce type de satire par l'attachement pathologique de l'archichancelier à la mise en scène de soi, qui l'a placé dans un espace de représentation - donc de critique - de type curial, où privé et public s'interpénètrent. N'aurait-il pas affirmé à Napoléon, pour justifier cette mise en scène de soi : "Les Français aiment à voir leurs princes, cela leur plaît et leur fait respecter le pouvoir $»^{38}$ ? Couvert de décorations, dont le grand cordon de la Légion d'honneur, "dispensateur de faveurs et de places $»^{39}$, l'archichancelier dominait sous l'Empire un réseau de clientèles et s'était entouré d'une véritable Cour. Il aimait à parader au Palais-Royal, dans des tenues anachroniques, en compagnie de ses amis de trente ans, les aristocrates montpelliérains d'Aigrefeuille et Villevieille, de son secrétaire particulier, Lavollée, et d'officiers de sa Maison. Habit à la française, perruque, jabot, manchettes en dentelles, cordons et médailles se prêtaient à la satire. La caricature « La promenade au Palais-Royal », publiée en mai 1814, rencontra ainsi un vif succès public ${ }^{40}$. Encore ne faisait-elle pas d'allusion claire au "petit défaut» de Cambacérès. La «Suite de la promenade au Palais-Royal » (fig. 54), publiée en juillet ${ }^{41}$, précise et sexualise la charge. Cambacérès n'est représenté qu'à travers son postérieur, alors qu'il se hisse dans une voiture - nouvelle métaphore de sa sortie de l'histoire et de la politique ${ }^{42}$. Le retournement du corps public en un corps privé, ici réduit à une paire de fesses, atteint son paroxysme. D'Aigrefeuille, au centre, lance un regard lubrique et tend son épée vers ledit postérieur. La scène est redoublée par un geste inconvenant

(37) N2 fol. Portraits. Parme (Jean-Jacques Régis de Cambacérès), vol. 1446. Le titre s'inspire de nouveau d'un vaudeville de Désaugiers, M. Lagobe, ou un tour de Carnaval, représenté au théâtre des Variétés en 1809. Lagobe, personnage ridicule, vient à Paris pour trouver une épouse, et tombe amoureux d'un mannequin exposé par un marchand de masques.

(38) Souvenirs du comte de Montgaillard, agent de la diplomatie secrète pendant la Révolution, l'Empire et la Restauration, publiés d'après des documents inédits par Clément de Lacroix, Paris, Paul Ollendorff, 1895, p. 256.

(39) Pierre Vialles, op. cit., p. 353.

(40) Journal de Paris, 30 mai 1814.

(41) « Il vient de paraître encore une caricature très indécente contre Camb... Il est représenté montant dans une voiture, et chatouillé par-derrière de la pointe de l'épée de M. d'Aig....» Bulletin de Paris (rapport quotidien du préfet de police au ministre de l'Intérieur) du 13 juillet 1814. A.N., $F^{7} 3836$.

(42) Plusieurs caricatures mettent en scène le « depart » de Cambacérès. 
de Villevieille, à droite, qui passe un doigt dans le croupion d'une poularde, et en lèche un autre. La promenade publique, mise en scène de Cour, devient la pitoyable exposition d'une déviance sexuelle partagée. Si l'on ajoute que le Palais-Royal était l'un des lieux privilégiés de la «subculture» sodomite du Paris du début $\mathrm{du} \mathrm{XIX}^{\mathrm{e}}$ siècle ${ }^{43}$, on saisit encore mieux la nature de la charge.

\section{Caricatures et imaginaires sociaux de la sodomie}

Les caricaturistes, au nom de la critique politique, usent le plus souvent d'un conformisme social. C'est là toute l'ambiguïté de l'image satirique, à la fois anticipatrice dans ses effets politiques, et conformiste voire traditionnaliste dans ses systèmes de représentations sociales. Les imaginaires sexuels auxquels se réfèrent ici les caricaturistes relèvent ainsi d'un artefact, d'une « culture populaire » en partie postulée. À ce titre, le corpus étudié n'autorise pas de conclusions générales sur les imaginaires sociaux de la sodomie, mais une réflexion sur les usages qui en sont faits, et sur la superposition de cadres mentaux d'âges différents, dans le cadre d'une campagne d'abord politique dans ses visées.

\section{Une pratique contre-nature}

Les caricaturistes s'inspirent parfois de représentations anciennes, non conformes à l'ordre juridique né de la Révolution, témoignant de la résistance de stéréotypes de longue durée. La laïcisation du droit sous la Révolution s'est accompagnée d'une "dépénalisation 》 de la sodomie, autrefois définie comme un crime contre Dieu ${ }^{44}$. Les codes pénaux de 1791 et de 1810 ne mentionnent ni la sodomie, ni la pédérastie; la répression des actes homosexuels passe désormais par le biais de l'" outrage public à la pudeur», ou, plus spécifiquement, de «la débauche ou la corruption de la jeunesse $»^{45}$. Or, la référence à l'incendie de Sodome, présente dans plusieurs caricatures, renvoie au châtiment anachronique

(43) Michael Sibalis, «The Palais-Royal and the Homosexual Subculture of NineteenthCentury Paris », dans Jeffrey Merrick et Michael Sibalis (éd.), Homosexuality in French History and Culture, New York, Harrington Park Press, 2001, p. 117-129.

(44) Cambacérès, contrairement à la légende, n'a d'ailleurs rien à voir avec cette dépénalisation. Voir notamment Michael Sibalis, "The Regulation of Male Homosexuality in Revolutionary and Napoleonic France, 1789-1815 », dans Jeffrey Merrick et Bryant T. Ragan (éd.), Homosexuality in Modern France, New York, Oxford University Press, 1996, p. 80-101.

(45) Respectivement articles 330 et 334 du Code pénal de 1810. 
du bûcher, pratiqué de plus en plus rarement au cours du XVIII ${ }^{\mathrm{e}}$ siècle ${ }^{46}$. La « fin du monde» (fig. 47 et fig. 48, cahier couleur), si elle ne relève pas nécessairement d'un imaginaire religieux, associe la sodomie à une perversion qui nie la visée procréative de la sexualité, érigée en loi naturelle.

Les représentations iconographiques de la sodomie comme acte contre-nature se conforment à un imaginaire commun, attesté par d'autres voies ${ }^{47}$. Contre-nature, le sodomite l'est en ce qu'il subvertit les frontières de genre. C'est tout le sens des caricatures de Cambacérès en travesti, « Tante Urlurette » (fig. 46, cahier couleur) ou « Un marchand de ridicules $»^{48}$. L'imitation homosexuelle de la féminité, nouvelle semble-t-il, participe de la « subculture » sodomite apparue au cours du XVIII ${ }^{\mathrm{e}}$ siècle $^{49}$, et envahit le champ des représentations au cours du XIX ${ }^{\mathrm{e}}$ siècle. On est là dans l'une des incarnations possibles du "troisième sexe $"^{50}$, à un moment - le début du $\mathrm{XIX}^{\mathrm{e}}$ siècle - où l'identité masculine reste fondée sur une virilité affirmée voire agressive ${ }^{51}$. Cette transgression est source de dégoût, largement partagé par les contemporains. Le dégoût provoqué par " l'infamie »- terme désignant la sodomie dans le discours policier à la fin du XVIII ${ }^{\mathrm{e}}$ siècle - trouve ici à s'incarner dans la figure du porc, dont on connaît les usages politiques par les caricaturistes révolutionnaires, principalement autour du Louis XVI de Varennes ${ }^{52}$. Au porc impur, glouton et sale s'ajoute désormais le cochon luxurieux et libidineux. Dans la Ménagerie de la rue impériale (fig. 55), Cambacérès est figé sous les traits d'un cochon, décrit par la légende comme un «pourceau du Gomore [sic] dont les goûts sont très bizarres ». Il se nourrit dans une auge pleine d'excréments, et hume de son groin le derrière d'un chien « aimant comme lui la bonne chair et mangeant à la même auge »

(46) Michel Rey, «Du péché au désordre : police et sodomie à Paris au XVIII ${ }^{\mathrm{e}}$ siècle », Revue d'histoire moderne et contemporaine, t. 29, janvier-mars 1982, p. 113-124.

(47) Thierry Pastorello, Sodome à Paris. Proto-histoire de l'homosexualité masculine, fin XVIII ${ }^{e}$-milieu XIX ${ }^{e}$ siècles, thèse de doctorat d'histoire sous la dir. d'André Gueslin, Univ. ParisDiderot, 2009, p. 202-214.

(48) « Le marchand de ridicules », BNF Estampes, coll. De Vinck, nº 9311.

(49) Thierry Pastorello, thèse citée, p. 31-32, 115-117.

(50) Laure Murat, op. cit., chapitre 1.

(51) André Rauch, Crise de l'identité masculine : 1789-1914, Paris, Hachette Littératures, 2000; Anne-Marie SoHn, "Sois un homme »! La construction de la masculinité au XIXe siècle, Paris, Le Seuil, 2009.

(52) Annie Duprat, Les rois de papier. La caricature de Henri III à Louis XVI, Paris, Belin, 2002, p. 227-232 et 237-244. Louis XVIII, de nouveau en 1814-1815, est également caricaturé en cochon. 
- on aura reconnu d'Aigrefeuille. On ne sera pas surpris de voir réunis dans ce bestiaire « impérial » les deux animaux successivement associés à la luxure dans l'imaginaire occidental ${ }^{53}$. On retrouve cet imaginaire excrémentiel de la sodomie dans une caricature cryptée, «la pêche du poisson d'avril » où Cambacérès et ses compères ne récoltent, en fait de poissons, que des excréments ${ }^{54}$. Le cochon apparait dans une autre caricature visant Cambacérès, «Le Plaisir » (fig. 56). Cette caricature de 1814 représente l'archichancelier exhibant son cordon rouge (celui de la Légion d'honneur, dont il était Grand-Aigle) et traînant un Cupidon à tête de porc et fesses énormes. Ce dernier s'appuie sur une fourchette embrochant une culotte et porte un clystère ${ }^{55}$. Il glisse à Cambacérès, bien sûr parderrière, un «plaisir »- du nom d'une pâtisserie d'origine médiévale orné d'abeilles impériales. Gourmandise et luxure sodomite se croisent de nouveau.

\section{Une contre-société}

La sodomie est montrée comme une pratique ou une conduite, plus que comme une identité. Le personnage de l'homosexuel n'est pas encore né, dont la médecine de la deuxième moitié du XIX ${ }^{\mathrm{e}}$ siècle cherchera les stigmates, aux frontières du physique et du moral ${ }^{56}$. Les caricaturistes suggèrent ici un dérèglement sexuel plus qu'une attirance exclusive pour le même sexe. Ce dérèglement est figuré par une pulsion scopique, qui porte le sujet sodomite à fixer son regard sur toute paire de fesses remarquable, qu'elle soit féminine ou masculine (fig. 53 et 54, par exemple)! Ce qui importe alors est la transgression du geste plus que la transgression du genre évoquée plus haut. La sodomie est rapportée à une sexualité purement hédonique et intrinsèquement perverse. Il n'est donc guère étonnant de voir se croiser, en caricature, les figures du « sodomite» Cambacérès et de la Vénus hottentote (« Le Jugement de Pâris », fig. 57). La Vénus hottentote, exhibée chez un montreur d'ani-

(53) Michel Pastoureau, Le cochon. Histoire d'un cousin mal aimé, Paris, Découvertes Gallimard, 2009, p. 98-100.

(54) «La pêche du poisson d'avril », BNF Estampes, coll. de Vinck, n 9312. La pêche fait sans doute allusion à l'attente désespérée de Cambacérès, en avril 1814, de se voir nommé pair de France par Louis XVIII.

(55) Fréquent dans la caricature révolutionnaire, le clystère renvoie d'ordinaire à l'idée de purgation politique. Ici, il souligne s'il était besoin l'allusion à la sodomie.

(56) Voir notamment Jean-Paul Aron et Roger KempF, Le pénis et la démoralisation de l'Occident, Paris, Le Livre de Poche, « Biblio essais », 1999. 
maux de la rue Saint-Honoré, non loin donc du Palais-Royal interlope de Cambacérès, avait éveillé une curiosité anthropologique mêlée de désir sexuel. Perçue comme un chaînon intermédiaire entre l'homme et l'animal, elle exposait contre rémunération son anatomie singulière, sa stéatopygie (une prolifération adipeuse au niveau des fesses) et sa macronymphie (l'élongation des lèvres inférieures du sexe, ou « tablier hottentot »), sources de tous les fantasmes ${ }^{57}$. Son arrivée à Paris, en septembre 1814, coïncide avec la campagne contre Cambacérès. La caricature montre Cambacérès avec ses deux compères habituels, comparant la Vénus hottentote (stéatopyge) à deux Vénus callipyges, et décernant, nouveau Pâris, une pomme aux plus belles fesses du trio! L'altérité sexuelle et l'altérité anthropologique sont habilement mêlées par le caricaturiste, qui entrecroise aussi deux actualités "mondaines» du Paris de la première Restauration.

Par ailleurs, les caricatures reproduisent l'imaginaire, très présent au XVIII ${ }^{\mathrm{e}}$ comme au XIX ${ }^{\mathrm{e}}$ siècles, d'une micro-société sodomite, libertine et élitaire. Elles se réfèrent ponctuellement, sans surprise, à des modèles antiques - Hadrien et Antinoüs (fig. 49, cahier couleur), ou l'" amour grec $\gg^{58}$. Cambacérès, Villevieille et d'Aigrefeuille, trio ordinairement croqué par les caricaturistes, appartiennent tous trois à l'aristocratie - Villevieille et d'Aigrefeuille sont tous deux marquis, Cambacérès, issu de la vieille noblesse de robe, est fait duc de Parme et prince de l'Empire en 1808. L'idée d'une aristocratie du vice est communément partagée à la fin $\mathrm{du} \mathrm{XVIII}{ }^{\mathrm{e}}$ siècle, et les sodomites sont fréquemment qualifiés de « chevaliers de la manchette ». En l'occurrence, Cambacérès porte sur les caricatures de très visibles manchettes à dentelles, ainsi que ses compagnons Villevieille et d'Aigrefeuille. Cet « ordre de la manchette » est perçu comme une contre-société, avec ses codes et ses rites initiatiques ${ }^{59}$. L'effet est ici renforcé par l'appartenance connue de Cambacérès - et de ses deux compères - à la franc-maçonnerie. Les rites initiatiques de cette confrérie sodomite sont ici suggérés par des jeux enfantins à forte connotation sexuelle : le jeu du cheval fondu (sorte de saute-mouton, fig. 58 et

(57) François-Xavier Fauvelle-Aymar, « Les tribulations de la Vénus hottentote », L'histoire, $\mathrm{n}^{\circ} 273$, février 2003, p. 80-84.

(58) «J'aime mieux un bon Louis que tous vos Napoléons ». BNF Estampes, Coll. de Vinck, $\mathrm{n}^{\circ}$ 9316. Sur cette estampe, on peut voir d'Aigrefeuille admirer un buste d'éphèbe, avec cette inscription : « l'amour grec ».

(59) Thierry Pastorello, thèse citée, p. 30. 
59) et de la main chaude ${ }^{60}$ (fig. 60). Les positions des uns et des autres et l'exhibition des fesses ne laissent pas de doute sur la nature sexuelle de la charge. On ajoutera que le graveur du « Jeu du cheval fondu » avait signé en bas : « Sodomia fecit amateur $»^{61}$.

L'idée d'une contagion sociale du vice, qui hante les entrepreneurs de morale au XVIII ${ }^{\mathrm{e}}$ comme au XIX ${ }^{\mathrm{e}}$ siècle, transparait également dans les caricatures. La relative tolérance sociale, au XVIII ${ }^{\mathrm{e}}$ siècle, à l'égard du libertinage aristocratique, y compris sodomite, ne s'étendait pas au reste de la société. Les domestiques occupent ainsi une place importante dans les victimes de la répression du « beau vice » au XVIII ${ }^{\mathrm{e}}$ siècle. Aussi le jeu sexuel entre le duc de Parme et son cuisinier (fig. 59) est-il sans doute apparu doublement scandaleux. Abandonnant ses instruments de cuisine - au premier plan - le jeune cuisinier exhibe son postérieur à l'archichancelier, pour un douteux jeu du cheval fondu. Le domestique - ici un cuisinier, car l'unité des caricatures repose sur la confusion des registres culinaire et sexuel - est entraîné dans la perversion par un maître amateur du « beau vice », conformément à l'imaginaire libertin du XVIII siècle - et, dans une certaine mesure, à des pratiques attestées ${ }^{62}$. Plus encore, le sodomite est perçu comme une menace potentielle pour la jeunesse, qu'il risque à tout moment de corrompre. Représentations communes et ordre juridique sont ici convergents : c'est à travers la « corruption de la jeunesse » (article 334 du Code pénal) que l'homosexualité est souvent réprimée au $\mathrm{XIX}^{\mathrm{e}}$ siècle. Le « jeu de la main chaude », représentant d'Aigrefeuille et Cambacérès au milieu d'un groupe de jeunes "mignons » figure une telle menace. Le « retour de la chasse aux culs blancs » y ajoute une allusion à des pratiques de prostitution masculine, dont on connaît le développement au $\mathrm{XIX}^{\mathrm{e}}$ siècle, notamment au Palais-Royal: d'Aigrefeuille et Villevieille ramènent leur "gibier » à Cambacérès armés d'un sac de « poudre d'or ».

\section{Dérision, exclusion et inconvenance}

Dans l'imaginaire du pouvoir, la sodomie fonctionne en ce début de Restauration comme l'équivalent de l'impuissance prêtée en son temps à Louis XVI. Elle renvoie aussi, comme tout dérèglement de mœurs, à l'idée

(60) Dans ce jeu, l'un des joueurs, courbé sur les genoux d'un autre, les yeux fermés, tend une main derrière lui, et doit deviner l'identité de celui qui la lui touche.

(61) A.N., BB ${ }^{18} 945$.

(62) Thierry Pastorello, thèse citée, p. 82. 
de corruption, cœur de la critique politique depuis la Révolution ${ }^{63}$. Elle discrédite, par les effets d'un imaginaire hybride, un serviteur de l'État de premier plan, à l'heure d'une possible reconversion politique. La transformation d'une rumeur en image publique a ainsi contribué à exclure Cambacérès du champ politique légitime. Exclusion confirmée, s'il en était besoin, par une caricature publiée en septembre 1814, la «Pompe funèbre de ma Tante Urlurette, ou il n'en faut plus parler ». On peut y voir deux cuisiniers porter le cadavre de Cambacérès à l'église, tandis que d'Aigrefeuille arrache d'un mur les caricatures - en abîme - visant le défunt. Un double message domine : la mort civile de l'ancien archichancelier est désormais effective, et la satire graphique n'y est pas étrangère.

\section{Une campagne ad hominem}

Cette satire s'inscrit dans une "guerre des images » impitoyable, favorisée par un régime de liberté relative de l'estampe - jusqu'en 1820, les caricatures sont soustraites à toute censure préalable ${ }^{64}$. Au début de la Restauration, en 1814-1815, les caricatures politiques visent essentiellement Napoléon - suscitant des résistances parfois violentes ${ }^{65}$ - et beaucoup plus marginalement les girouettes. D'autres « sodomites » supposés ou avérés, tels Barras ou Fiévée ${ }^{66}$, semblent épargnés par la satire politique. Aussi l'acharnement des caricaturistes à l'encontre de Cambacérès interroge-t-il. Un corpus aussi conséquent, singulier dans son registre, concentré dans le temps - quelques mois pour l'essentiel-, et redoublé par une écriture de l'anathème ${ }^{67}$, laisse entrevoir une campagne ad hominem, probablement orchestrée. Ce que certains contemporains dénoncèrent explicitement, notamment le propre neveu de Cambacérès :

(63) Robert Darnton indique que le thème dominant des 82 Vies privées satiriques publiées entre 1789 et 1802 concerne la corruption (Le diable dans un bénitier [...], op. cit., p. 559). Lynn Hunt observe quant à elle les liens entre débauche et corruption politique dans la pornographie politique sous la Révolution (The Invention of Pornography [...], op. cit., p. 308).

(64) Robert Justin Goldstein, Censorship of Political Caricature in Nineteenth-Century France, Kent, Kent State University Press, 1989, p. 100.

(65) Les rapports de police mentionnent ainsi des caricatures anti-napoléoniennes menacées voire déchirées par des soldats démobilisés. A.N., $F^{7} 3733$ et 3836 .

(66) Sur Fiévée, préfet de la Nièvre, et « homosexuel » affirmé, voir Jean Tulard, Joseph Fiévée conseiller secret de Napoléon, Paris, Fayard, 1985, p. 164-165.

(67) Si nous n'avons pas relevé de pamphlets visant exclusivement Cambacérès, des traces d'incrimination politique peuvent être trouvées dans des textes polémiques et des chansons, tel cet extrait du Champ de mai, à propos de la fin du gouvernement de Napoléon en 1815 : «Cambacérès poliment / Compose un beau compliment / Les douceurs de Son Altesse / Font un effet surprenant / L'assemblée incontinent / S'esquive en serrant les fesses » (L'Ami des Bourbons, ou chansonnier des royalistes, Paris, Le Normant, 1815, p. 46). 
«Les pamphlétaires et les faiseurs de caricatures aux ordres de la police furent alors presque exclusivement déchainés contre lui; mais chacun sait aujourd'hui que cet acharnement, dont $M$. le duc de Cambacérès a pu être glorieux, n'était que le résultat d'une manœuvre, employée avec succès, pour éloigner de la direction des affaires les personnes dont on redoutait l'influence. Dans cette intention, M. le duc de Cambacérès a été l'un des plus maltraités, et il avait le droit de l'être. Les meneurs de l'époque sacrifièrent tout au besoin de le rendre ridicule, à défaut d'autres moyens, pour le faire descendre de la scène politique, et l'empêcher ensuite d'y reparaître, parce qu'il était devenu ridicule $»^{68}$.

Les commanditaires d'une telle campagne sont difficiles à identifier au sein des cercles du pouvoir. Les caricatures sont pour la plupart anonymes, elles ne sont pas déclarées à la Direction de la Librairie ${ }^{69}$, et les rares noms de caricaturistes, d'imprimeurs et de libraires identifiés ${ }^{70}$ ne permettent pas de reconstituer un réseau de responsables. L'identité « ultraroyaliste » d'un des marchands d'estampes impliqués dans cette campagne $^{71}$ constitue un indice intéressant mais non suffisant. Un personnage sulfureux figure aussi parmi de possibles commanditaires : le comte de Montgaillard, ancien agent secret du comte de Provence puis de Napoléon, rallié aux Bourbons, publiciste prolifique et très zélé, explicitement évoqué par le neveu de Cambacérès ${ }^{72}$.

Le mobile, quant à lui, est clairement définissable : écarter de l'espace public le second de Napoléon, potentielle girouette. Les rapports de police de la première Restauration témoignent d'une attention maniaque des autorités aux faits et gestes de l'archichancelier ${ }^{73}$, et de la crainte constante du retour sur scène d'un «transformiste » politique. Son silence intrigue, perçu comme une conversion de façade au nouvel

(68) «Observations au sujet des imputations contenues dans les Mémoires de M. de Bourrienne sur l'archichancelier ", in Bourrienne et ses erreurs volontaires ou involontaires, ou observations sur ses mémoires, par AB, Paris, Heideloff, 1830, p. 34-35.

(69) En dépit de ce qu'affirme la lettre de certaines caricatures. A.N., $F^{18}(\mathrm{VI}) 2$. Déclaration des imprimeurs. Estampes, année 1814.

(70) Grâce au procès que nous évoquerons infra.

(71) Il s'agit de Beaublé, quai des Grands Augustins. «Ce Beaublé est un bon royaliste, dit-on, mais de ces royalistes emportés dans leurs opinions et qui vont quelquefois trop loin pour la tranquillité publique » (Bulletin de Paris du 27 octobre 1814. A.N., F 3837).

(72) «Son acharnement [de Montgaillard] vient de ce que [Cambacérès] refusa de conclure le marché honteux qu'il lui fit proposer, et qui consistait à acheter son silence moyennant quelques milliers de francs » (Bourrienne et ses erreurs [...] op. cit., p. 30).

(73) 13 bulletins de police (sur 68) sur «l'esprit public» à Paris mentionnent ainsi Cambacérès entre le 7 juillet et le 15 septembre 1814 . A.N., $F^{7} 3836$. 
ordre des choses. Le préfet de police rapporte avec inquiétude les subtils propos qu'aurait tenus Cambacérès en août 1814 :

« Je ne vois personne, je n'ai été ni chez M. le comte d'Artois, arrivé le premier, ni chez les autres princes. Je n'ai point été voir le roi. Ainsi, si des événements arrivaient, je suis encore tout aussi près des puissants de la terre que j'en ai été; et de toute part, je n'ai à encourir aucun reproche; car enfin la Cour ne peut me reprocher ma réserve, et si Bonaparte ou la Régence survenait, je n'aurais point contre moi ma soumission et mes révérences $»^{74}$.

Son silence prudent, selon les autorités, serait destiné à induire « que s'il était rattaché à la chose actuelle, il la servirait bien, puisqu'il ne fait rien pour l'arrêter $»^{75}$. Cette disponibilité de Cambacérès, réelle ou non, a probablement renforcé la détermination de certains royalistes à l'éliminer symboliquement. Ancien conventionnel, Cambacérès, quoiqu'ayant voté le sursis, est assimilé à la catégorie des régicides, et honni à ce titre par les plus intransigeants des royalistes.

Effets et paradoxes de la satire sexuelle

Une certitude : l'exposition des caricatures chez les marchands d'estampes, au Palais-Royal, sur les boulevards, sur les quais de Seine ou rue Saint-Jacques, produit des effets politiques. Cambacérès se terre dans son hôtel particulier, réduit son personnel de maison, n'ose plus, en octobre 1814 « se montrer en public pendant le jour ». " Le soir, il se [promène] sur le boulevard du Temple, seul ou suivi d'un domestique sans livrée $»^{76}$. Il est vrai que quelques mois plus tôt, le 4 juillet 1814 , il « a été reconnu se promenant sur le boulevard du Temple avec les personnes qui l'accompagnent ordinairement. Suivi pendant quelque moment et bientôt entouré par des gardes du corps en uniforme, il a reçu force quolibets et allait être insulté plus gravement encore si quelques passants n'eussent protégé sa retraite jusqu'à sa voiture $\gg^{77}$. Les sources taisent la nature et l'origine des insultes. On ne peut prouver avec certitude la responsabilité des caricatures dans cette montée des violences. Toutefois, la coïncidence

(74) Bulletin de Paris du 30 août 1814. A.N., F 3836.

(75) Bulletin de Paris du 22 août 1814. A.N., F 3836.

(76) Bulletin de Paris du 18 octobre 1814. A.N., F 3837.

(77) Bulletin de Paris, 7 juillet 1814. A.N., F 3836. 
dans le temps ${ }^{78}$ et dans l'espace ${ }^{79}$ avec la publication des estampes les plus virulentes contre l'archichancelier, ne peut manquer de troubler.

Le langage des caricaturistes, s'il est politiquement opportun, a en effet franchi le seuil de l'inconvenance morale. Les visées politiques du message ont été brouillées par un contenu fortement sexualisé. La représentation, même allusive, de la déviance était en soi intolérable. Les archives judiciaires et policières démontrent avec certitude le désaccord d'une partie des autorités avec la nature de la campagne graphique visant Cambacérès. Il s'agit parfois, tout simplement, de protéger le frère de Cambacérès, archevêque de Rouen, de la flétrissure morale infligée à l'archichancelier. Aussi les caricatures en cause sont-elles saisies dans le département de la Seine-Inférieure, sur ordre du préfet ${ }^{80}$. Mais plus généralement, le directeur général de la police, Beugnot, ancien préfet napoléonien, et le garde des Sceaux, Dambray, royaliste modéré, sont tous deux intervenus pour faire saisir, dans le lot des caricatures hostiles à Cambacérès, celles qui leur semblaient contraires à «l'honnêteté publique». Dambray dénonce un « sujet de scandale pour les pères de famille vertueux, pour les étrangers et même pour les Anglais, tout familiarisés qu'ils sont avec ce genre de productions $»^{81}$. Il souligne la nécessité d'une police des caricatures, qui respecte, quelle que soit la cible visée, les « bonnes mœurs » :

«Peu importe, sans doute, que ces caricatures attaquent principalement des personnages qui ont joué un grand rôle dans le gouvernement détruit; un gouvernement légitime n'a pas besoin de pareils auxiliaires et abstraction faite de toute autre considération, personne n'a le droit de rappeler ce que le roi oublie et pardonne. Rien au monde ne peut légitimer d'ailleurs des outrages publics faits aux bonnes mœurs $»^{82}$.

De ce plaidoyer semble découler implicitement un nouveau code de civilité propre à protéger les hommes publics de tout dévoilement, vrai ou faux, de leur vie privée. Code pour l'heure théorique, avant qu'il ne soit formalisé en 1819 par la régulation de la diffamation, à l'occasion de la loi de Serre sur la presse.

(78) C'est au même moment, en effet, que sont engagées des poursuites contre plusieurs caricatures jugées obscènes visant Cambacérès.

(79) Les Grands Boulevards étaient l'un des centres d'exposition des estampes et caricatures en ce début de $\mathrm{XIX}^{\mathrm{e}}$ siècle.

(80) Bulletin de police du 29 juin 1814. A.N., F 3733.

(81) Lettre du garde des Sceaux Dambray au procureur général près la cour royale de Paris, le 19 septembre 1814 . A.N., BB ${ }^{18} 945$.

(82) Ibid. 
Le procès, après une procédure d'appel encouragée par le garde des Sceaux, conduit à la saisie des caricatures les plus explicitement sexualisées, «Le Plaisir» (fig. 56), « Le cheval fondu » (fig. 58), « Le jeu du cheval fondu » (fig. 59), « La petite loge » (fig. 53), et « La main chaude » (fig. 60), et à la condamnation à des amendes d'un marchand d'estampes, de trois graveurs et de deux imprimeurs ${ }^{83} \ldots$ Un marchand d'estampes impliqué dans l'affaire comme diffuseur réagit alors en tenant «les propos les plus violents sur les autorités constituées qu'il accuse d'être les continuateurs de Bonaparte. Il se propose de faire une pétition au roi contre la police qui a saisi les caricatures $»^{84}$. Plus généralement, les autorités de police se plaignent de l'existence d'un procès public, regrettant que les caricaturistes et marchands d'estampes n'aient pas été « punis administrativement ${ }^{85}-$ sans débat public. Autant d'indices qui nous ramènent à l'orchestration politique, dans l'ombre, d'une campagne graphique révélée par le procès. Quant à Cambacérès lui-même, la victime de cette campagne, il semblerait, si l'on en croit des propos rapportés par la police, s'être accommodé de ces caricatures comme d'une manifestation inéluctable de la critique politique, sur le modèle anglais :

« On rapporte que M. Cambacérès a dit à quelqu'un qui lui parlait du procès des marchands de caricatures qu'il fallait laisser ces marchands libres de vendre les objets de critique dont il était question, qu'il n'y avait que les sots ou les timides qui s'en plaignaient, et qu'en cela il était sage d'imiter les Anglais qui ne se fâchaient point de se voir caricaturés $\rangle^{86}$.

À ses yeux, le rétablissement de l'honneur et de la dignité passe par l'écriture de soi. Face à la satire graphique, il envisage donc à l'automne 1814 de rédiger ses mémoires, pour « répandre du jour sur les événements de sa carrière politique $»^{87}$.

(83) Ibid. Le procès se tient en appel le 21 octobre 1814.

(84) Il s'agit de Beaublée, cité plus haut. Bulletin de Paris du 27 octobre 1814. A.N., F ${ }^{7}$ 3837.

(85) Bulletin de Paris du 2 septembre 1814 : « On aurait désiré que ces marchands eussent été punis administrativement, car le public commençait à tourner cette affaire en plaisanterie ». A.N., $\mathrm{F}^{7} 3836$.

(86) Bulletin de Paris du 15 septembre 1814. A.N., F 3836.

(87) Archives privées de Cambacérès, citées par Laurence ChATEL DE BrANCION, op. cit., p. 596. 
Cambacérès est écarté de toute fonction publique, sous la première comme sous la seconde Restauration. Après les Cent-Jours et son éphémère retour comme ministre de la Justice de Napoléon, il doit quitter le territoire, menacé par la « loi d'amnistie » du 12 janvier 1816, et s'exile donc à Bruxelles jusqu'en 1818.

La satire graphique a légitimé l'exclusion politique subie par Cambacérès. Les calembours sexuels, les regards lubriques et les exhibitions de postérieurs ne relèvent pas seulement du rire grivois ou de la satire mondaine. La présence, subliminale ou explicite, de la sodomie dans ces caricatures a eu des effets politiques. La dignité d'un homme d'État susceptible de s'adapter au nouvel ordre des choses a été profondément atteinte. À la façon des libelles et des «Vies privées », les caricatures ont fonctionné comme des dispositifs politiques tout en ne parlant pas - ou peu - de politique ${ }^{88}$. Elles ont multiplié les « anecdotes »-au sens d'histoires secrètes dévoilées au public -, se renvoyant les unes aux autres, et ont percé le « mur » d'une vie privée par ailleurs fantasmée. Ce mur qu'un contemporain, le doctrinaire Royer-Collard, souhaitait infranchissable dans un gouvernement représentatif ${ }^{89}$.

Pour saisir la violence symbolique de ces images, il importait alors de restituer l'historicité des imaginaires convoqués ${ }^{90}$. À cet égard, la sodomie suggérée ou montrée ne désigne pas seulement une déviance, ni même une corruption, mais une souillure et une menace pour l'ordre public, et à ce titre discrédite en profondeur celui qui, quelques semaines auparavant, exerçait une portion du pouvoir exécutif.

Emmanuel FurEIX

Université Paris Est - Créteil

Centre de recherches en histoire européenne comparée (CRHEC) 61 av. du général de Gaulle

94010 Créteil cedex

fureix@u-pec.fr

(88) Robert DARNTON, op. cit., p. 565-573.

(89) Lors des débats préparatoires à la loi de Serre de 1819, à propos de la diffamation, il a énoncé la maxime selon laquelle « la vie privée doit être murée » (d'après M. CHAssan, Traité des délits et contraventions de la parole, Paris, Videcoq, 1837, p. 336-337).

(90) Malgré certaines continuités, l'imaginaire présent dans les caricatures homophobes $\mathrm{du} \mathrm{xx}^{\mathrm{e}}$ siècle est assez différent. Florence TAMAGNE, «Caricatures homophobes et stéréotypes de genre en France et en Allemagne : la presse satirique de 1900 au milieu des années 1930 », Le temps des médias, 2003, $\mathrm{n}^{\circ}$ 1, p. $42-53$. 